PROCEEDINGS OF THE

AMERICAN MATHEMATICAL SOCIETY

Volume 132, Number 5, Pages 1483-1493

S 0002-9939(03)07217-4

Article electronically published on October 3, 2003

\title{
SPECTRAL SUBSPACES OF SUBSCALAR AND RELATED OPERATORS
}

\author{
T. L. MILLER, V. G. MILLER, AND M. M. NEUMANN
}

(Communicated by Joseph A. Ball)

\begin{abstract}
For a bounded linear operator $T \in L(X)$ on a complex Banach space $X$ and a closed subset $F$ of the complex plane $\mathbb{C}$, this note deals with algebraic representations of the corresponding analytic spectral subspace $X_{T}(F)$ from local spectral theory. If $T$ is the restriction of a generalized scalar operator to a closed invariant subspace, then it is shown that $X_{T}(F)=E_{T}(F)=$ $\bigcap_{\lambda \notin F}(\lambda-T)^{p} X$ for all sufficiently large integers $p$, where $E_{T}(F)$ denotes the largest linear subspace $Y$ of $X$ for which $(\lambda-T) Y=Y$ for all $\lambda \in \mathbb{C} \backslash F$. Moreover, for a wide class of operators $T$ that satisfy growth conditions of polynomial or Beurling type, it is shown that $X_{T}(F)$ is closed and equal to $E_{T}(F)$.
\end{abstract}

\section{INTRODUCTION AND BASIC DEFINITIONS}

If $T \in L(X)$ is a bounded normal operator with spectral measure $\mathfrak{E}$ on a complex Hilbert space $X$, then the ranges of the spectral projections enjoy the representation

$$
\operatorname{ran} \mathfrak{E}(F)=\bigcap_{\lambda \in \mathbb{C} \backslash F}(\lambda-T) X
$$

for every closed set $F \subseteq \mathbb{C}$; see [10, 1.5.7]. This remarkable description of the spectral measure in purely algebraic terms was obtained by Putnam [15] and, with a completely different approach, by Pták and Vrbová 13] after a slightly weaker result due to Johnson [8]. The formula is a basic tool in the theory of automatic continuity for linear transformations that intertwine a pair of normal operators; see 8] and [16]. Among other things, the result also leads, as observed by Whitley [18], to an elegant short proof of Fuglede's theorem on operators commuting with a normal one; see [10] 1.5.8].

In this note, we are concerned with similar results for the spectral subspaces associated with a bounded linear operator $T \in L(X)$ on a complex Banach space $X$; see [3] and [10]. For each closed set $F \subseteq \mathbb{C}$, the analytic spectral subspace $X_{T}(F)$ is defined to be the set of all $x \in X$ for which there exists a local resolvent function at each point $\lambda \in \mathbb{C} \backslash F$; that is, $x \in X_{T}(F)$ if and only if every $\lambda \in \mathbb{C} \backslash F$ has an open neighborhood $V$ and an analytic function $f: V \rightarrow X$ such that $(\mu-T) f(\mu)=x$ for all $\mu \in V$. These linear subspaces, while generally not closed, play a fundamental role in the spectral theory of operators on Banach spaces. It is a classical fact that, if $T$ is a normal operator, or, more generally, a spectral operator in the sense of

Received by the editors August 22, 2002 and, in revised form, January 14, 2003.

2000 Mathematics Subject Classification. Primary 47A11; Secondary 47B37, 47B40. 
Dunford, with spectral measure $\mathfrak{E}$, then $X_{T}(F)=\operatorname{ran} \mathfrak{E}(F)$ for every closed set $F \subseteq \mathbb{C}$; see [10, 1.2.25].

Moreover, the operator $T \in L(X)$ is said to have the single-valued extension property (SVEP) provided that, for each closed set $F \subseteq \mathbb{C}$ and every $x \in X_{T}(F)$, there is a globally defined analytic local resolvent function $f: \mathbb{C} \backslash F \rightarrow X$ such that $(\mu-T) f(\mu)=x$ for all $\mu \in \mathbb{C} \backslash F$. It is well known that (SVEP) is equivalent to the condition that $X_{T}(\emptyset)=\{0\}$; see [10, 1.2.16 and 3.3.2]. A sufficient condition for (SVEP) is Dunford's property $(C)$, namely the requirement that $X_{T}(F)$ be closed for every closed set $F \subseteq \mathbb{C}$; see [10, 1.2.19 and 3.3.4].

Associated with the operator $T$ and each closed subset $F$ of $\mathbb{C}$ is also an algebraic spectral subspace $E_{T}(F)$, defined to be the linear span of the collection of all (not necessarily closed) linear subspaces $Y$ of $X$ for which

$$
(\lambda-T) Y=Y \text { for each } \lambda \in \mathbb{C} \backslash F \text {; }
$$

see [10, 1.4]. Evidently, $E_{T}(F)$ is the largest linear subspace $Y$ for which $(\lambda-T) Y$ $=Y$ for all $\lambda \in \mathbb{C} \backslash F$. These spaces, with an equivalent definition, were introduced in [9] in connection with certain problems in automatic continuity. It follows from [10, 1.2.16] that $X_{T}(F) \subseteq E_{T}(F)$ for every $T \in L(X)$ and closed set $F \subseteq \mathbb{C}$. Thus, if $T$ has no nontrivial divisible subspace in the sense that $E_{T}(\emptyset)=\{0\}$, then clearly $T$ has (SVEP). Moreover, for every closed set $F \subseteq \mathbb{C}$, we obtain the chain of inclusions

$$
X_{T}(F) \subseteq E_{T}(F) \subseteq \bigcap_{\lambda \in \mathbb{C} \backslash F}(\lambda-T)^{p} X,
$$

valid for every integer $p \geq 0$. Consequently, if these two inclusions hold as identities for some integer $p \geq 0$, then the corresponding identities hold for every integer $q \geq p$.

The representation formula for normal operators mentioned above was extended to hyponormal operators by Clancey [2] Thm. 1.3.5]; specifically, if $T$ is hyponormal, then

$$
X_{T}(F)=E_{T}(F)=\bigcap_{\lambda \in \mathbb{C} \backslash F}(\lambda-T) X
$$

for all closed sets $F \subseteq \mathbb{C}$. In particular, it follows that the intersection of the ranges $(\lambda-T) X$ over all $\lambda \in \mathbb{C}$ is trivial when $T$ is hyponormal; see [2, Cor. 1.3.3]. By contrast, a cohyponormal operator satisfies this condition only when it is normal; see [2, Cor. 1.4.1].

An important generalization of the normal operators to the setting of Banach spaces is the class of generalized scalar operators; see [3] and [10]. An operator $T \in L(X)$ is generalized scalar provided that there exists a continuous algebra homomorphism $\Phi: \mathcal{E}(\mathbb{C}) \rightarrow L(X)$ for which $\Phi(1)=I$ and $\Phi(z)=T$, where, as usual, $\mathcal{E}(\mathbb{C})$ denotes the Fréchet algebra of all infinitely differentiable complexvalued functions on $\mathbb{C}$. Vrbová [17] showed that, essentially, the structure of the spectral subspaces of normal operators extends to the case of generalized scalar operators: if $T \in L(X)$ is generalized scalar, then

$$
X_{T}(F)=E_{T}(F)=\bigcap_{\lambda \in \mathbb{C} \backslash F}(\lambda-T)^{p} X
$$

for all sufficiently large integers $p$ and all closed sets $F \subseteq \mathbb{C}$; see also [4], [7], and [10, 1.4.14, 1.4.15, 1.5.4, and 1.5.5] for further related results. 
Since generalized scalar operators have (SVEP) ([10, 1.4.10]), Vrbová's result shows, in particular, that generalized scalar operators have no divisible subspace different from zero. On the other hand, there are simple examples of well-behaved operators with nontrivial divisible subspaces. For instance, as noted in [10, 1.4.12 and 1.5.6], the classical Volterra operator $T$ on the space $X=C([0,1])$ is both compact and quasi-nilpotent, admits a nonanalytic functional calculus, and satisfies $X_{T}(\emptyset)=\{0\}$, but $E_{T}(\emptyset)=\bigcap_{n \in \mathbb{N}} T^{n} X \neq\{0\}$.

While much of the previous work focused on the case of operators with good spectral decomposition properties, certain examples suggest a broader context for the representation of the spectral subspaces.

For instance, if $T$ is a semi-shift on a nonzero Banach space $X$, that is, if $T$ is an isometry for which $\bigcap_{n \in \mathbb{N}} T^{n} X=\{0\}$, then $X_{T}(F) \neq\{0\}$ if and only if $F$ contains $\sigma(T)$, the closed unit disc; see [10, 1.6.8]. Since, by [10 1.6.2], the approximate point spectrum of $T$ is the unit circle $\mathbb{T}$, a basic result on the Kato spectrum ([10. 3.1.11]) entails that $\bigcap_{\lambda \in U}(\lambda-T) X=X_{T}(\mathbb{T})=\{0\}$ for every nonempty open subset $U$ of the open unit disc. From this it is immediate that the representation $X_{T}(F)=E_{T}(F)=\bigcap_{\lambda \notin F}(\lambda-T) X$ holds for all closed $F \subseteq \mathbb{C}$ in the semi-shift case as well. In fact, Yoo $[19,2.7]$ recently proved that every Banach space isometry $T$ satisfies $X_{T}(F)=E_{T}(F)$ for all closed $F \subseteq \mathbb{C}$.

An operator $T \in L(X)$ is said to be subscalar provided that $T$ is similar to the restriction of a generalized scalar operator to a closed invariant subspace. Both hyponormal operators and isometries are subscalar (11] and [10, 1.6.6, 1.6.7, and 2.4.9]), and so, in light of Clancey and Yoo's results, it is natural to ask if subscalar operators inherit the representation of spectral subspaces of generalized scalar operators. In this note, we apply a construction of Eschmeier and Putinar (5] or [6] 6.4]) to show that this is actually the case. We also establish that, for a remarkably large class of operators defined by certain growth conditions, the analytic and algebraic spectral subspaces coincide. The results are exemplified in the case of unilateral weighted right shifts.

\section{TOOLS FROM LOCAL SPECTRAL THEORY}

We refer the reader to [6, 6.4 and Appendix 4] or [10, 2.3] for a complete account of the spaces considered in this section. For an open subset $V$ of $\mathbb{C}$ and an arbitrary Banach space $X$, let $L^{2}(V, X)$ denote the Banach space of $X$-valued measurable functions on $V$ that are Bochner square-integrable with respect to area measure $\mu$ on $V$. Denote the canonical norm on $L^{2}(V, X)$ by $\|\cdot\|_{2, V}$.

For every integer $k \geq 0$, define the Sobolev-type space

$$
W^{k}(V, X):=\left\{f \in L^{2}(V, X): \bar{\partial}^{j} f \in L^{2}(V, X) \text { for } j=0,1, \ldots, k\right\},
$$

where the derivatives $\bar{\partial}^{j} f$ with respect to $\bar{z}$ are understood in the sense of distributions. Then $W^{k}(V, X)$ is a Banach space with respect to the norm given by

$$
\|f\|_{W^{k}(V, X)}^{2}:=\sum_{j=0}^{k}\left\|\bar{\partial}^{j} f\right\|_{2, V}^{2} .
$$

We also consider the Fréchet space $\mathcal{E}(V, X)$ of infinitely differentiable vectorvalued functions, with topology generated by uniform convergence of all orders of derivatives $D^{\alpha} f:=\partial_{x}^{\alpha_{1}} \partial_{y}^{\alpha_{2}} f$, where $\alpha=\left(\alpha_{1}, \alpha_{2}\right) \in \mathbb{N}_{0}^{2}$, on the compact subsets of 
$V$. Analogous to the classical Sobolev embedding theorem, we have, in an obvious sense,

$$
\mathcal{E}(V, X)=\bigcap\left\{W^{k}(U, X): k \geq 0 \text { and } U \text { is open and relatively compact in } V\right\},
$$

and the Fréchet space topology on $\mathcal{E}(V, X)$ is, in fact, generated by the seminorms given by $\left\|\bar{\partial}^{k} f\right\|_{2, U}$, where $k \geq 0$ and $U$ is open with compact closure in $V$; see [10, 2.3.7]. Moreover, by [6, A4.3], every compactly supported $f \in W^{k}(V, X)$ can be approximated by functions in $\mathcal{D}(V, X)$, the space of functions in $\mathcal{E}(V, X)$ with compact support contained in $V$.

The following lemma follows from [10, 2.3.9] and the vector-valued analogue of the proof of [10, 2.3.5].

Lemma 1. If $V \subseteq \mathbb{C}$ is a bounded open set and $k \geq 0$, then $\bar{\partial}: W^{k+1}(V, X) \rightarrow$ $W^{k}(V, X)$ is surjective. Also, for every $\varphi \in \mathcal{D}(V, X)$, the Cauchy transform of $\varphi$ given by

$$
\widehat{\varphi}(z):=\int_{V} \frac{\varphi(\zeta)}{\zeta-z} \mathrm{~d} \mu(\zeta) \quad \text { for all } z \in \mathbb{C}
$$

belongs to $\mathcal{E}(\mathbb{C}, X)$ and satisfies $-\frac{1}{\pi} \bar{\partial} \widehat{\varphi}=\varphi$. Moreover, there is a constant $c>0$, independent of $k$, so that

$$
\|\widehat{\varphi}\|_{W^{k+1}(V, X)} \leq c\|\varphi\|_{W^{k}(V, X)}
$$

for all $\varphi \in \mathcal{D}(V, X)$. Finally, if $\varphi=\bar{\partial} \psi$ for some $\psi \in \mathcal{D}(V, X)$, then $\psi=-\frac{1}{\pi} \widehat{\varphi}$.

Proof. By the Cauchy-Pompeiu formula,

$$
\psi(z)=-\frac{1}{\pi} \int_{V} \frac{\bar{\partial} \psi(\zeta)}{\zeta-z} d \mu(\zeta)
$$

for all $\psi \in \mathcal{D}(V, X)$ and $z \in V$. In particular, the last statement of the lemma holds. Moreover, if $\varphi \in \mathcal{D}(V, X)$ and $h \in \mathcal{D}(V)$, then, by Fubini's theorem,

$$
\begin{aligned}
\int_{V}\left(-\frac{1}{\pi} \widehat{\varphi}(z)\right) \bar{\partial} h(z) d \mu(z) & =-\frac{1}{\pi} \int_{V} \int_{V} \frac{\varphi(\zeta) \bar{\partial} h(z)}{\zeta-z} d \mu(\zeta) d \mu(z) \\
& =\frac{1}{\pi} \int_{V} \int_{V} \frac{\varphi(\zeta) \bar{\partial} h(z)}{z-\zeta} d \mu(z) d \mu(\zeta) \\
& =-\int_{V} \varphi(\zeta) h(\zeta) d \mu(\zeta),
\end{aligned}
$$

and so $-\frac{1}{\pi} \bar{\partial} \widehat{\varphi}=\varphi$ in the sense of distributions. If $g(z):=-z^{-1}$ for all nonzero $z \in V-V$ and $g(0):=0$, then $\widehat{\varphi}=g * \varphi$ on $V$, and a standard convolution estimate implies that $\|\hat{\varphi}\|_{2, V} \leq\|g\|_{1, V-V}\|\varphi\|_{2, V}$. Thus

$$
\begin{aligned}
\|\hat{\varphi}\|_{W^{k+1}(V, X)}^{2} & =\|\widehat{\varphi}\|_{2, V}^{2}+\|\bar{\partial} \hat{\varphi}\|_{W^{k}(V, X)}^{2} \\
& \leq\|g\|_{1, V-V}^{2}\|\varphi\|_{2, V}^{2}+\pi^{2}\|\varphi\|_{W^{k}(V, X)}^{2} \\
& \leq\left(\|g\|_{1, V-V}^{2}+\pi^{2}\right)\|\varphi\|_{W^{k}(V, X)}^{2}
\end{aligned}
$$

for every $\varphi \in \mathcal{D}(V, X)$.

Let $T \in L(X)$ be an arbitrary operator on a Banach space $X$. As usual, we denote by $\sigma(T)$ the spectrum of $T$ and by $\rho(T)$ the resolvent, $\rho(T):=\mathbb{C} \backslash \sigma(T)$. For $f \in \mathcal{E}(V, X)$, we define $T_{V} f$ to be the function given by $T_{V} f(z):=(z-T) f(z)$. 
Clearly, the operator $T_{V}$ is continuous on each $W^{k}(V, X)$ and $\mathcal{E}(V, X)$. The quotient space $\mathcal{E}(\mathbb{C}, X) / \overline{T_{\mathbb{C}} \mathcal{E}(\mathbb{C}, X)}$ is a Fréchet space with generating seminorms given by

$$
\rho_{k, V}\left(f+\overline{T_{\mathbb{C}} \mathcal{E}(\mathbb{C}, X)}\right):=\inf \left\{\left\|f+T_{V} g\right\|_{W^{k}(V, X)}: g \in \mathcal{E}(\mathbb{C}, X)\right\}
$$

where $V$ ranges over the bounded open neighborhoods of $\sigma(T)$ and $k \geq 0$.

Lemma 2. Suppose that $T \in L(X)$, let $k \geq 0$ and $V$ be a bounded open set containing $\sigma(T)$, and define $Y_{k, V}:=W^{k}(V, X) / \overline{T_{V} W^{k}(V, X)}$. Then the mapping

$$
i_{k, V}: \mathcal{E}(\mathbb{C}, X) / \overline{T_{\mathbb{C}} \mathcal{E}(\mathbb{C}, X)} \rightarrow Y_{k, V}
$$

given by $i_{k, V}\left(f+\overline{T_{\mathbb{C}} \mathcal{E}(\mathbb{C}, X)}\right):=f+\overline{T_{V} W^{k}(V, X)}$ for all $f \in \mathcal{E}(\mathbb{C}, X)$ is welldefined, and the seminorm on $\mathcal{E}(\mathbb{C}, X) / \overline{T_{\mathbb{C}} \mathcal{E}(\mathbb{C}, X)}$ given by

$$
\nu_{k, V}\left(f+\overline{T_{\mathbb{C}} \mathcal{E}(\mathbb{C}, X)}\right):=\left\|i_{k, V}\left(f+\overline{T_{\mathbb{C}} \mathcal{E}(\mathbb{C}, X)}\right)\right\|_{Y_{k, V}}
$$

for all $f \in \mathcal{E}(\mathbb{C}, X)$ is equivalent to $\rho_{k, V}$. Specifically, there is a constant $c>0$ such that

$$
\nu_{k, V}([f]) \leq \rho_{k, V}([f]) \leq c \nu_{k, V}([f])
$$

for every $[f] \in \mathcal{E}(\mathbb{C}, X) / \overline{T_{\mathbb{C}} \mathcal{E}(\mathbb{C}, X)}$. Moreover, if $f \in \mathcal{D}(V, X)$ and $\nu_{k, V}([f])=0$, then there is a sequence $\left(\varphi_{n}\right)_{n \in \mathbb{N}}$ in $\mathcal{D}(V, X)$ such that $\left\|f-T_{V} \varphi_{n}\right\|_{W^{k}(V, X)} \rightarrow 0$ as $n \rightarrow \infty$.

Proof. If $f+\overline{T_{\mathbb{C}} \mathcal{E}(\mathbb{C}, X)}=g+\overline{T_{\mathbb{C}} \mathcal{E}(\mathbb{C}, X)}$ for some $f, g \in \mathcal{E}(\mathbb{C}, X)$, then there exists a sequence $\left(g_{n}\right)_{n \in \mathbb{N}}$ in $\mathcal{E}(\mathbb{C}, X)$ such that $T_{\mathbb{C}} g_{n} \rightarrow f-g$ in $\mathcal{E}(\mathbb{C}, X)$ and so, in particular, $\left\|T_{V} g_{n}-(f-g)\right\|_{W^{k}(V, X)} \rightarrow 0$ by [10, 2.3.7]. Thus

$$
f+\overline{T_{V} W^{k}(V, X)}=g+\overline{T_{V} W^{k}(V, X)}
$$

in $Y_{k, V}$, and the mapping $i_{k, V}: \mathcal{E}(\mathbb{C}, X) / \overline{T_{\mathbb{C}} \mathcal{E}(\mathbb{C}, X)} \rightarrow Y_{k, V}$ is well-defined.

Clearly, $\rho_{k, V}([f]) \geq \nu_{k, V}([f])$. To establish the remaining estimate, we argue as in the proof of [5] 4.5]. Suppose that $\left(f_{n}\right)_{n \in \mathbb{N}}$ is a sequence in $\mathcal{E}(\mathbb{C}, X)$ for which $\nu_{k, V}\left(\left[f_{n}\right]\right) \rightarrow 0$. Let $\theta \in \mathcal{E}(\mathbb{C})$ be such that $\theta \equiv 0$ in a neighborhood of $\sigma(T)$ and $1-\theta \in \mathcal{D}(V)$. If $\left(g_{n}\right)_{n \in \mathbb{N}}$ is chosen in $W^{k}(V, X)$ such that $\left\|f_{n}-T_{V} g_{n}\right\|_{W^{k}(V, X)} \rightarrow 0$, then

$\theta(z)(z-T)^{-1} f_{n}(z)+(1-\theta(z)) g_{n}(z)-g_{n}(z)=\theta(z)\left((z-T)^{-1} f_{n}(z)-g_{n}(z)\right) \rightarrow 0$ in $W^{k}(V, X)$. Since $(1-\theta) g_{n} \in W^{k}(V, X)$ has compact support, we may choose a sequence $\left(h_{n}\right)_{n \in \mathbb{N}}$ in $\mathcal{D}(V, X)$ so that $\left\|(1-\theta) g_{n}-h_{n}\right\|_{W^{k}(V, X)} \rightarrow 0$. If

$$
\varphi_{n}(z):=\theta(z)(z-T)^{-1} f_{n}(z)+h_{n}(z),
$$

then $\varphi_{n} \in \mathcal{E}(\mathbb{C}, X)$, and $\left\|f_{n}-T_{V} \varphi_{n}\right\|_{W^{k}(V, X)} \rightarrow 0$. Thus $\rho_{k, V}\left(\left[f_{n}\right]\right) \rightarrow 0$ whenever $\nu_{k, V}\left(\left[f_{n}\right]\right) \rightarrow 0$; in particular, $\rho_{k, V}([f])=0$ whenever $\nu_{k, V}([f])=0$ in the space $\mathcal{E}(\mathbb{C}, X) / \overline{T_{\mathbb{C}} \mathcal{E}(\mathbb{C}, X)}$. To show the existence of a constant $c>0$ such that $\rho_{k, V}([f]) \leq c \nu_{k, V}([f])$ for every $[f]$, suppose otherwise. Then there is a sequence $\left(f_{n}\right)_{n \in \mathbb{N}}$ in $\mathcal{E}(\mathbb{C}, X)$ such that $\rho_{k, V}\left(\left[f_{n}\right]\right)>n \nu_{k, V}\left(\left[f_{n}\right]\right)$ for each $n \in \mathbb{N}$. It follows that $\nu_{k, V}\left(\left[f_{n}\right]\right)>0$ for every $n$, and so we may define $g_{n}:=\left(n \nu_{k, V}\left(\left[f_{n}\right]\right)\right)^{-1} f_{n}$ in $\mathcal{E}(\mathbb{C}, X)$ for each $n \in \mathbb{N}$. Then $\nu_{k, V}\left(\left[g_{n}\right]\right) \rightarrow 0$ as $n \rightarrow \infty$, while $\rho_{k, V}\left(\left[g_{n}\right]\right)>1$ for every $n \in \mathbb{N}$, a contradiction.

Finally, notice that the argument above shows that whenever $f \in \mathcal{D}(V, X)$ and $\nu_{k, V}([f])=0$, then the functions $\varphi_{n}(z):=\theta(z)(z-T)^{-1} f(z)+h_{n}(z)$ are each in $\mathcal{D}(V, X)$ and satisfy $\left\|f-T_{V} \varphi_{n}\right\|_{W^{k}(V, X)} \rightarrow 0$. 
If $k \geq n$ and if $V$ and $U$ are bounded open sets for which $V \supseteq U \supseteq \sigma(T)$, then the restriction mapping from $W^{k}(V, X)$ into $W^{n}(U, X)$ induces a mapping from $Y_{k, V}$ into $Y_{n, U}$, and $i_{n, U}$ factors through $Y_{k, V}$. Thus the previous lemma implies that $\mathcal{E}(\mathbb{C}, X) / \overline{T_{\mathbb{C}} \mathcal{E}(\mathbb{C}, X)}$ is the projective limit of the spaces $Y_{k, V}$, where $k$ is a nonnegative integer and $V$ is a bounded open neighborhood of $\sigma(T)$.

A computation shows that, for every $f \in \mathcal{E}(\mathbb{C})$, the multiplication operator given by $M_{f} g(z):=f(z) g(z)$ for all $g \in W^{k}(V, X)$ is bounded on each $W^{k}(V, X)$, and that the mapping $f \mapsto M_{f}$ is continuous with respect to the sub-multiplicative seminorm given by

$$
\sigma_{k, \bar{V}}(f):=\sum_{\alpha \in \mathbb{N}_{0}^{2},|\alpha| \leq k} \frac{1}{\alpha !} \sup \left\{\left|D^{\alpha} f(z)\right|: z \in \bar{V}\right\}
$$

([10, page 57]), and thus extends continuously to $C^{k}(\mathbb{C})$, the Fréchet algebra of all $k$-times continuously differentiable functions on $\mathbb{C}$; see $10,2.4 .1$ and 2.4.2]. Letting $M_{z}$ denote multiplication by the independent variable, it follows that $M_{z} \in$ $L\left(W^{k}(V, X)\right)$ is generalized scalar of order at most $k$. Define $S_{k, V}$ on $Y_{k, V}$ to be the operator $S_{k, V}=\left[M_{z}\right]$ for each $k \geq 0$ and bounded open set $V$ containing $\sigma(T)$. Then $S_{k, V}$ is a generalized scalar operator with functional calculus given by $\Phi(f):=\left[M_{f}\right]$ for all $f \in \mathcal{E}(\mathbb{C})$, and $S_{k, V}$ also has order $\leq k$. Moreover, $\rho(T) \subseteq \rho\left(S_{k, V}\right)$. Indeed, if $A \in L(X)$ commutes with $T$, then $A[f]:=[A f]$ yields a well-defined continuous linear mapping on $Y_{k, V}$ that commutes with $S_{k, V}$. In particular, $S_{k, V}[f]=T[f]$, and, if $\lambda \in \rho(T)$, then $\left(\lambda-S_{k, V}\right)(\lambda-T)^{-1}=(\lambda-T)^{-1}\left(\lambda-S_{k, V}\right)=I$. By [3, 1.6] or [10, 1.4.10], $\sigma\left(S_{k, V}\right)$ is the support of the homomorphism $\Phi$, that is, the smallest closed set $F$ for which $\Phi(f)=0$ whenever $\operatorname{supp}(f) \cap F=\emptyset$. Thus, when considering operators $\left[M_{f}\right]$ on $Y_{k, V}$, we may assume that $f \in \mathcal{D}(V)$.

If, for every open set $V \subseteq \mathbb{C}$, the corresponding continuous mapping $T_{V}$ on $\mathcal{E}(V, X)$ is a topological monomorphism, that is, if $T_{V}: \mathcal{E}(V, X) \rightarrow \mathcal{E}(V, X)$ is injective and has closed range, then $T$ is said to have property $(\beta)_{\mathcal{E}}$. The significance of this property is that it is an intrinsic characterization of subscalarity; see [5, 4.5] or [6 6.4.8]. Notice that for $T$ to have property $(\beta)_{\mathcal{E}}$ it is enough to check that $T_{\mathbb{C}}$ is injective and with closed range. Indeed, if $\left(f_{n}\right)_{n \in \mathbb{N}}$ is a sequence in $\mathcal{E}(V, X)$ such that $T_{V} f_{n} \rightarrow 0$ in $\mathcal{E}(V, X)$ and if $K$ is a compact subset of $V$, choose $\varphi \in \mathcal{D}(V)$ such that $\varphi \equiv 1$ in an open neighborhood of $K$. Then $T_{\mathbb{C}}\left(\varphi f_{n}\right) \rightarrow 0$ in $\mathcal{E}(\mathbb{C}, X)$ and so, by assumption, $\varphi f_{n} \rightarrow 0$ in $\mathcal{E}(\mathbb{C}, X)$. It follows that $\sup _{K}\left\|\bar{\partial}^{j} f_{n}\right\| \rightarrow 0$ for every $j \geq 0$, and, since $K$ was arbitrary, that $f_{n} \rightarrow 0$ in $\mathcal{E}(V, X)$.

Suppose that $T \in L(X)$ is subscalar, equivalently, that $T$ has property $(\beta)_{\mathcal{E}}$. Then $T_{\mathbb{C}} \mathcal{E}(\mathbb{C}, X)$ is closed in $\mathcal{E}(\mathbb{C}, X)$, and, as observed in the proof of [5, 4.5], the mapping $J: X \rightarrow \mathcal{E}(\mathbb{C}, X) / T_{\mathbb{C}} \mathcal{E}(\mathbb{C}, X)$ given by $J(x):=[x]$ is a topological monomorphism. Therefore, by the open mapping theorem, there exist an integer $n \geq 0$, a bounded open set $V_{0} \subseteq \mathbb{C}$ containing $\sigma(T)$, and a constant $c>0$ so that $\|x\| \leq c \rho_{n, V_{0}}(J x)$ for every $x \in X$. Clearly then, for every $x \in X$,

$$
\|x\| \leq c \rho_{k, V}(J x),
$$

whenever $k \geq n$ and $V \supseteq V_{0}$. Moreover, by Lemma [2] the mapping $J_{k, V}:=i_{k, V} J$ is bounded below and intertwines $T$ and $S_{k, V}$, and so each $S_{k, V} \in L\left(Y_{k, V}\right)$ is a generalized scalar extension of $T$.

Proposition 3. Suppose that $T \in L(X)$ is subscalar and that $n \in \mathbb{N}$ and $V_{0}$ is a bounded open set containing $\sigma(T)$ so that there exists a $c>0$ for which $\|x\| \leq$ 
$c \rho_{n, V_{0}}(J x)$ for all $x \in X$. Then, for every $x \in X$ and every closed set $F \subseteq \mathbb{C}$,

$$
J_{n, V_{0}}(x) \in Y_{S_{n, V_{0}}}(F) \Leftrightarrow J_{k, V}(x) \in Y_{S_{k, V}}(F)
$$

for every integer $k \geq n$ and every bounded open set $V$ that contains $V_{0}$.

Proof. Let $k \geq n$ and $V \subseteq \mathbb{C}$ be a bounded open set such that $V_{0} \subseteq V$. Then, by [10, 1.4.10] and the observation following Lemma 2 ,

$$
Y_{S_{k, V}}(F)=\left\{[g]:\left[M_{f}\right][g]=0 \text { for all } f \in \mathcal{D}\left(V_{0}\right) \text { such that } \operatorname{supp}(f) \cap F=\emptyset\right\} .
$$

If $J_{k, V_{0}}(x) \in Y_{S_{k, V_{0}}}(F)$ and $\varphi \in \mathcal{D}\left(V_{0}\right)$ is $\operatorname{such}$ that $\operatorname{supp}(\varphi) \cap F=\emptyset$, then, by Lemma 2, there is a sequence $\left(\varphi_{n}\right)_{n \in \mathbb{N}}$ in $\mathcal{D}\left(V_{0}, X\right)$ such that

$$
\left\|\varphi x-T_{V} \varphi_{n}\right\|_{W^{k}(V, X)}=\left\|\varphi x-T_{V_{0}} \varphi_{n}\right\|_{W^{k}\left(V_{0}, X\right)} \rightarrow 0 .
$$

Thus $J_{k, V_{0}}(x) \in Y_{S_{k, V_{0}}}(F)$ implies that $J_{k, V}(x) \in Y_{S_{k, V}}(F)$ whenever $V \supseteq V_{0}$.

It remains to be seen that if $J_{k, V}(x) \in Y_{S_{k, V}}(F)$, then $J_{k+1, V}(x) \in Y_{S_{k+1, V}}(F)$. If $J_{k, V}(x) \in Y_{S_{k, V}}(F)$ and $\varphi \in \mathcal{D}(V \backslash F)$, then $[\varphi x]=0$ and $[\bar{\partial} \varphi x]=0$ in $Y_{k, V}$. Thus, by Lemma 2, there are sequences $\left(\varphi_{n}\right)_{n \in \mathbb{N}}$ and $\left(\psi_{n}\right)_{n \in \mathbb{N}}$ in $\mathcal{D}(V, X)$ so that $\varphi x-T_{V} \varphi_{n} \rightarrow 0$ and $-\frac{1}{\pi} \bar{\partial} \varphi x-T_{V} \psi_{n} \rightarrow 0$ in $W^{k}(V, X)$. By Lemma 1

$$
\varphi x-\widehat{T_{V} \psi_{n}}=\left(-\frac{1}{\pi} \bar{\partial} \varphi x-T_{V} \psi_{n} \widehat{)} \rightarrow 0\right.
$$

in $W^{k+1}(V, X)$. But, if $x_{n}:=\int_{V} \psi_{n} d \mu$, then $x_{n} \in X$ and

$$
\widehat{T_{V} \psi_{n}}(z)=\int_{V} \frac{(\zeta-T) \psi_{n}(\zeta)}{\zeta-z} d \mu(\zeta)=\int_{V} \frac{(\zeta-z+z-T) \psi_{n}(\zeta)}{\zeta-z} d \mu(\zeta) ;
$$

hence $\widehat{T_{V} \psi_{n}}(z)=x_{n}+T_{V} \widehat{\psi}_{n}(z)$ for all $z \in V$. Since $\varphi x-\left(x_{n}+T_{V} \widehat{\psi}_{n}\right) \rightarrow 0$ in $W^{k+1}(V, X)$ and $\varphi x-T_{V} \varphi_{n} \rightarrow 0$ in $W^{k}(V, X)$, it follows that

$$
\left\|J_{k, V} x_{n}\right\|_{Y_{k, V}} \leq\left\|x_{n}-T_{V}\left(\varphi_{n}-\widehat{\psi}_{n}\right)\right\|_{W^{k}(V, X)} \rightarrow 0 .
$$

But $J_{k, V}$ is bounded below. So $x_{n} \rightarrow 0$ in $X$ and therefore $\varphi x-T_{V} \widehat{\psi}_{n} \rightarrow 0$ in $W^{k+1}(V, X)$. Thus $[\varphi x]=0$ in $Y_{k+1, V}$. Since $\varphi \in \mathcal{D}(V \backslash F)$ was arbitrary, it follows that $J_{k+1, V}(x) \in Y_{S_{k+1, V}}(F)$.

\section{MAin Results}

As a consequence of the results of Section 2, we may now extend Vrbová's characterization of the spectral subspaces of generalized scalar operators to subscalar operators.

Theorem 4. For every subscalar operator $T \in L(X)$ on a Banach space $X$, there exists an integer $p \in \mathbb{N}$ such that

$$
X_{T}(F)=E_{T}(F)=\bigcap_{\lambda \in \mathbb{C} \backslash F}(\lambda-T)^{p} X \quad \text { for all closed sets } F \subseteq \mathbb{C} .
$$

Proof. With the notation and results of the preceding section, choose an integer $n \in \mathbb{N}$ and a bounded open neighborhood $V_{0}$ of $\sigma(T)$ such that there exists a constant $c>0$ for which $\|x\| \leq c \rho_{n, V_{0}}(J x)$ for every $x \in X$, and let $p \geq n+3$.

If $F \subseteq \mathbb{C}$ is closed, then, by [10, 1.2.16], $X_{T}(F) \subseteq E_{T}(F) \subseteq \bigcap_{\lambda \notin F}(\lambda-T)^{p} X$, and so we need only show that $\bigcap_{\lambda \notin F}(\lambda-T)^{p} X \subseteq X_{T}(F)$. Let $x \in \bigcap_{\lambda \notin F}(\lambda-T)^{p} X$ 
be given. Since $S_{n, V_{0}}$ is generalized scalar of order $\leq n$, it follows from [10 1.5.4] that

$$
J_{n, V_{0}}(x) \in J_{n, V_{0}}\left(\bigcap_{\lambda \notin F}(\lambda-T)^{p} X\right) \subseteq \bigcap_{\lambda \notin F}\left(\lambda-S_{n, V_{0}}\right)^{p} Y_{n, V_{0}}=Y_{S_{n, V_{0}}}(F),
$$

and therefore, by Proposition [3, $J_{k, V}(x) \in Y_{S_{k, V}}(F)$ for every $k \geq n$ and every bounded open set $V$ for which $V \supseteq V_{0}$.

Now, suppose that $W \subseteq \mathbb{C}$ is a bounded open set such that $\bar{W} \cap F=\emptyset$. Choose $\varphi \in \mathcal{E}(\mathbb{C})$ such that $\varphi \equiv 1$ on $\bar{W}$ and $\operatorname{supp}(\varphi) \cap F=\emptyset$. Then $J_{k, V}(x) \in Y_{S_{k, V}}(F)$ implies, again by [10,1.4.10], that $[\varphi x]=0$ in $Y_{k, V}$ for every $k \geq n$ and $V \supseteq V_{0}$. Therefore, by the equivalence of the seminorms $\rho_{k, V}$ and $\nu_{k, V}$ from Lemma 2 we have $\rho_{k, V}([\varphi x])=0$ for each $k \geq n$ and $V \supseteq V_{0}$; that is, $[\varphi x]=0$ in $\mathcal{E}(\mathbb{C}, X) / T_{\mathbb{C}} \mathcal{E}(\mathbb{C}, X)$. Thus there is a function $g \in \mathcal{E}(\mathbb{C}, X)$ such that $\varphi(z) x=(z-T) g(z)$ for every $z \in \mathbb{C}$. In particular,

$$
x=(z-T) g(z) \quad \text { for all } z \in W .
$$

Applying $\bar{\partial}$, we obtain the identities

$$
0=\bar{\partial} x=\bar{\partial}((z-T) g(z))=(z-T) \bar{\partial} g(z) \quad \text { for all } z \in W .
$$

But the mapping $T_{W}$ on $\mathcal{E}(W, X)$ is injective, because $T$ has property $(\beta)_{\mathcal{E}}$, and so $\bar{\partial} g \equiv 0$ in $\mathcal{E}(W, X)$. Thus $g: W \rightarrow X$ is an analytic function satisfying $T_{W} g=x$, and therefore $x \in X_{T}(\mathbb{C} \backslash W)$. Since $\mathbb{C} \backslash F=\bigcup\{W: W$ bounded and open with $\bar{W} \cap F=\emptyset\}$, it follows that $x \in X_{T}(F)$, as desired.

As indicated by the preceding proof, the representation formula of Theorem 4 holds for every integer $p$ for which $p \geq n+3$, whenever $n \in \mathbb{N}, V_{0}$ is a bounded open set containing $\sigma(T)$, and there exists a $c>0$ for which $\|x\| \leq c \rho_{n, V_{0}}(J x)$ for every $x \in X$. The sufficiency of the exponent $n+3$ in the case of generalized scalar operators was first obtained by Foiaş and Vasilescu, 7, 3.1 and 3.2], who also showed that, for certain generalized scalar operators of order $n=0$, the exponent $p=2$ is, in fact, the best lower bound; see [7, 2.10 and 4.4].

If $T \in L(X)$ is subscalar, then the actual values of $n$ and $V_{0}$ for which $J_{n, V_{0}}$ is bounded below are unknown; their existence is simply a consequence of the open mapping theorem for Fréchet spaces. Therefore, in practice, Theorem 4 establishes the algebraic description of $X_{T}(F)$ only for exponents $p$ sufficiently large. Hyponormal operators all have a generalized scalar extension of order 2 (14]) and obviously subnormal operators have an order 0 extension. But even if it were known that the extension $S_{n, V_{0}} \in L\left(Y_{n, V_{0}}\right)$ were of order 0 , we could obtain the representation only for $p \geq 3$. Thus Clancey's result that $p=1$ suffices for hyponormal operators is strictly stronger.

We finally turn to the algebraic representation of the analytic spectral subspaces for operators that satisfy certain growth conditions. For an arbitrary operator $T \in L(X)$ on a nonzero Banach space $X$, let $\kappa(T):=\inf \{\|T x\|:\|x\|=1\}$ denote the lower bound of $T$. Clearly, $\kappa(T)=\left\|T^{-1}\right\|^{-1}$ provided that $T$ is invertible.

As in [11, an operator $T \in L(X)$ is said to satisfy Beurling's condition $(B)$ if

$$
\sum_{n=1}^{\infty} \frac{1}{n^{2}}\left(\left|\log \kappa\left(T^{n}\right)\right|+\left|\log \left\|T^{n}\right\|\right|\right)<\infty .
$$


A classical result of Colojoară and Foiaş ([3, 5.3.2] or [10, 4.4.7]) confirms that every invertible operator with property $(B)$ is decomposable and, in fact, superdecomposable, meaning that, for every open cover $\{U, V\}$ of $\mathbb{C}$, there exists an operator $R \in L(X)$ commuting with $T$ such that $\sigma(T \mid \overline{R(X)}) \subseteq U$ and $\sigma(T \mid \overline{(I-R)(X)})$ is contained in $V$; see [10, 1.4.1].

Evidently, property $(B)$ is inherited by restrictions to nonzero closed invariant subspaces, but it seems to be an interesting open problem whether every operator with property $(B)$ admits an invertible extension with property $(B)$; see $[11$ for certain partial results. In fact, it is not known if every operator with property $(B)$ is similar to the restriction of a decomposable operator to a closed invariant subspace, equivalently, if Beurling's property $(B)$ implies Bishop's property $(\beta)$; see 10 , 1.2.5 and 2.4.4]. The following result contains a partial answer.

Theorem 5. Let $T \in L(X)$ be an operator that satisfies Beurling's condition $(B)$, and suppose that $T$ has no divisible subspace different from zero. Then $T$ has Dunford's property $(C)$, and the identity $X_{T}(F)=E_{T}(F)$ holds for every closed set $F \subseteq \mathbb{C}$.

Proof. Clearly, condition $(B)$ on $T$ implies that $\kappa\left(T^{n}\right)^{1 / n} \rightarrow 1$ and $\left\|T^{n}\right\|^{1 / n} \rightarrow 1$ as $n \rightarrow \infty$, and therefore, by [10 1.6.2], that the approximate point spectrum $\sigma_{\text {ap }}(T)$ is contained in the unit circle. Thus, again by [10,1.6.2], $\sigma(T)$ is either the entire closed unit disc or contained in the unit circle.

Next observe that, by [10, 1.4.13], the assertion of the theorem means precisely that the space $E_{T}(F)$ is closed for every closed set $F \subseteq \mathbb{C}$. Because $E_{T}(F)=$ $E_{T}(F \cap \sigma(T))$, it suffices to verify that $E_{T}(F)$ is closed for an arbitrary closed set $F \subseteq \mathbb{C}$ that does not contain the open unit disc $\mathbb{D}$. We may also assume that $Y:=\overline{E_{T}(F)}$ is nonzero, so that $S:=T \mid Y \in L(Y)$ inherits property $(B)$ from $T$.

Now choose a point $\lambda \in \mathbb{D} \backslash F$, and note that $(\lambda-T) E_{T}(F)=E_{T}(F)$. Since $\lambda \in \mathbb{D}$ and therefore $\lambda \notin \sigma_{\text {ap }}(T)$, the operator $\lambda-T$ is bounded below. This implies that $(\lambda-T) Y$ is closed, and hence $(\lambda-S) Y=Y$. Since $\lambda-S$ inherits injectivity from $\lambda-T$, we infer that $\lambda \notin \sigma(S)$, which shows that $\sigma(S)$ does not contain $\mathbb{D}$. By the dichotomy for the spectrum of operators with property $(B)$ mentioned above, we conclude that $S$ is an invertible operator with property $(B)$, and hence, by [10,4.4.7], that $S$ is super-decomposable. On the other hand, because $E_{S}(\emptyset) \subseteq E_{T}(\emptyset)=\{0\}$, the operator $S$ has no nontrivial divisible subspace. Thus, by [10 1.4.14], $E_{S}(F)$ coincides with $Y_{S}(F)$, and hence is closed. Since it is easy to check that $E_{T}(F)=E_{S}(F)$, we see that $E_{T}(F)$ is closed, as desired.

In the preceding result, it is essential to require that $T$ has no nontrivial divisible subspace, since, by [10, 1.4.13], the space $E_{T}(\emptyset)$ is closed only when it is zero. However, Theorem [5 can be improved under a stronger growth condition. As in [11], we say that $T \in L(X)$ satisfies the polynomial growth condition $(P)$ if there exist $c, s>0$ such that

$$
\frac{1}{c n^{s}} \leq \kappa\left(T^{n}\right) \leq\left\|T^{n}\right\| \leq c n^{s} \quad \text { for all } n \in \mathbb{N} .
$$

Evidently, $(P)$ implies $(B)$. Moreover, if $T$ is invertible, then, by another classical result due to Colojoară and Foiaş, [3, 5.1] or [10, 1.5.12], $T$ has property $(P)$ precisely when $T$ is generalized scalar with $\sigma(T) \subseteq \mathbb{T}$. Thus every nontrivial restriction of a generalized scalar operator with spectrum in the unit circle satisfies condition 
$(P)$, but the converse is open in general. In fact, it seems to be unknown if $(P)$ implies $(\beta)$. Nevertheless, we obtain the following counterpart to Theorem 4

Theorem 6. Every operator $T \in L(X)$ with property $(P)$ has Dunford's property $(C)$, and satisfies $X_{T}(F)=E_{T}(F)$ for every closed set $F \subseteq \mathbb{C}$.

Proof. Evidently, one may proceed as in the proof of Theorem 5 to conclude that $S$ is an invertible operator with property $(P)$, and therefore generalized scalar. Thus we may refer to Vrbová's result [17] to see that $E_{S}(F)=Y_{S}(F)$ without having to require that $E_{T}(\emptyset)=\{0\}$. The argument is then completed as above.

We conclude with a few applications of the preceding results. First, representation formulas of the type presented in Theorem 4 have, by [10, 1.4.16], interesting consequences for the behavior of the local resolvent functions and their derivatives. For instance, as noted by Bermúdez and González [1, Clancey's result [2, Thm. 1.3.5] entails that, for every hyponormal operator for which the spectrum has empty interior, all nontrivial local resolvent functions have to be unbounded; see also [10] 1.5.9].

Second, operators that satisfy the conclusion of Theorems 5 and 6 are, by [10. 1.4.13], precisely the admissible operators introduced in [12]. These operators play a natural role in the automatic continuity theory for linear transformations $A$ : $X \rightarrow Y$ that intertwine a pair of Banach space operators $T \in L(X)$ and $S \in L(Y)$ in the sense that $S A=A T$. Clearly, if $A$ is continuous, then $A X_{T}(F) \subseteq Y_{S}(F)$ for all closed sets $F \subseteq \mathbb{C}$, but this inclusion does not hold in general; see [10, 5.4.10]. If, however, $Y_{S}(F)=E_{S}(F)$, then, obviously, $A X_{T}(F) \subseteq A E_{T}(F) \subseteq E_{S}(F) \subseteq Y_{S}(F)$, even when the intertwiner $A$ is discontinuous; see [10] and [12] for further pertinent information.

For another illustration, we close with an example on unilateral weighted right shifts.

Corollary 7. Let $\left(\omega_{n}\right)_{n \in \mathbb{N}_{0}}$ be a bounded sequence of strictly positive real numbers, and let $T \in L(X)$ denote the corresponding unilateral weighted right shift on the sequence space $X:=\ell^{q}\left(\mathbb{N}_{0}\right)$ for some $1 \leq q<\infty$. Then the following assertions hold:

(a) if there exist constants $c, s>0$ such that

$$
\frac{1}{c n^{s}} \leq \inf _{k \geq 0} \omega_{k} \cdots \omega_{n+k-1} \leq \sup _{k \geq 0} \omega_{k} \cdots \omega_{n+k-1} \leq c n^{s} \quad \text { for all } n \in \mathbb{N},
$$

then $T$ is subscalar, and, for all sufficiently large integers $p$,

$$
X_{T}(F)=E_{T}(F)=\bigcap_{\lambda \in \mathbb{C} \backslash F}(\lambda-T)^{p} X \quad \text { for every closed set } F \subseteq \mathbb{C} ;
$$

(b) if there exist constants $c>0$ and $0<s<1$ such that

$$
\frac{1}{c \mathrm{e}^{n^{s}}} \leq \inf _{k \geq 0} \omega_{k} \cdots \omega_{n+k-1} \leq \sup _{k \geq 0} \omega_{k} \cdots \omega_{n+k-1} \leq c \mathrm{e}^{n^{s}} \quad \text { for all } n \in \mathbb{N},
$$

then $T$ is subdecomposable, and $X_{T}(F)=E_{T}(F)$ for every closed set $F \subseteq \mathbb{C}$.

Proof. From 11 we know that $T$ is subscalar in case $(a)$ and subdecomposable in case $(b)$. The assertions are now immediate from Theorems 4 and 5 together with the fact that unilateral weighted right shifts have no divisible subspace different from $\{0\}$. 
Added in proof. Vladimir Müller has recently shown (private communication) that every operator satisfying the growth condition $(B)$, respectively $(P)$, has an invertible extension that also satisfies $(B)$, respectively $(P)$. Thus property $(B)$ implies property $(\beta)$, and property $(P)$ characterizes $\mathcal{E}(\mathbb{T})$-subscalarity.

\section{REFERENCES}

[1] T. Bermúdez and M. González, On the boundedness of the local resolvent function, Integral Equations Operator Theory 34 (1999), 1-8. MR 2000b:47010]

[2] K. Clancey, Seminormal Operators, Lecture Notes in Math. 742, Springer-Verlag, New York, 1979. MR 81c: 47002

[3] I. Colojoară and C. Foiaş, Theory of Generalized Spectral Operators, Gordon and Breach, New York, 1968. MR 52:15085

[4] P. C. Curtis and M. M. Neumann, Nonanalytic functional calculi and spectral maximal spaces, Pacific J. Math. 137 (1989), 65-85. MR 90h:47059

[5] J. Eschmeier and M. Putinar, Bishop's condition $(\beta)$ and rich extensions of linear operators, Indiana Univ. Math. J. 37 (1988), 325-348. MR 89k:47051

[6] J. Eschmeier and M. Putinar, Spectral Decompositions and Analytic Sheaves, Clarendon Press, Oxford, 1996. MR 98h:47002

[7] C. Foiaş and F.-H. Vasilescu, Nonanalytic local functional calculus, Czechoslovak Math. J. 24 (99) (1974), 270-283. MR 51:8885

[8] B. E. Johnson, Continuity of linear operators commuting with continuous linear operators, Trans. Amer. Math. Soc. 128 (1967), 88-102. MR 35:4748

[9] B. E. Johnson and A. M. Sinclair, Continuity of linear operators commuting with continuous linear operators II, Trans. Amer. Math. Soc. 146 (1969), 533-540. MR 40:4791

[10] K. B. Laursen and M. M. Neumann, An Introduction to Local Spectral Theory, Clarendon Press, Oxford, 2000. MR 2001k:47002

[11] T. L. Miller, V. G. Miller, and M. M. Neumann, Growth conditions and decomposable extensions, Contemp. Math., 321 (2003), 197-205.

[12] M. M. Neumann, Decomposable operators and generalized intertwining linear transformations, Operator Theory: Advances and Applications 28 (1988), 209-222. MR 89f:47049

[13] V. Pták and P. Vrbová, On the spectral function of a normal operator, Czechoslovak Math. J. 23 (98) (1973), 615-616. MR 49:1196

[14] M. Putinar, Hyponormal operators are subscalar, J. Operator Theory 12 (1984), 385-395. MR 85h:47027

[15] C. R. Putnam, Ranges of normal and subnormal operators, Michigan Math. J. 18 (1971), 33-36. MR 43:2550

[16] A. M. Sinclair, Automatic Continuity of Linear Operators, London Math. Soc. Lecture Note Series 21, Cambridge University Press, Cambridge, 1976. MR 58:7011

[17] P. Vrbová, Structure of maximal spectral spaces of generalized scalar operators, Czechoslovak Math. J. 23 (98) (1973), 493-496. MR 47:9338

[18] R. Whitley, Fuglede's commutativity theorem and $\bigcap R(T-\lambda)$, Canad. Math. Bull. 33 (1990), 331-334. MR 92f:47017

[19] J.-K. Yoo, Local spectral theory for operators on Banach spaces, Far East J. Math. Sci., Special Volume (2001), Part III, 303-311. MR 2002m:47005

Department of Mathematics and Statistics, Mississippi State University, PO Drawer MA, Mississippi State, Mississippi 39762

E-mail address: miller@math.msstate.edu

Department of Mathematics and Statistics, Mississippi State University, PO Drawer MA, Mississippi State, Mississippi 39762

E-mail address: vivien@math.msstate.edu

Department of Mathematics and Statistics, Mississippi State University, PO Drawer MA, Mississippi State, Mississippi 39762

E-mail address: neumann@math.msstate.edu 